Relations industrielles

Industrial Relations

\title{
La socialisation
}

\section{Gérard Dion}

Volume 18, numéro 2, avril 1963

URI : https://id.erudit.org/iderudit/1021433ar

DOI : https://doi.org/10.7202/1021433ar

Aller au sommaire du numéro

Éditeur(s)

Département des relations industrielles de l’Université Laval

ISSN

0034-379X (imprimé)

1703-8138 (numérique)

Découvrir la revue

Citer ce document

Dion, G. (1963). La socialisation. Relations industrielles / Industrial Relations, 18(2), 265-269. https://doi.org/10.7202/1021433ar
Résumé de l'article

Quand on parle de « socialisation " on suscite immédiatement des réactions diverses, selon la tournure d'esprit, les passions, les intérêts de ceux à qui on s'adresse. C'est que le terme " socialisation " recèle des ambiguïtés qui sont sources d'embarras et de confusions. Il importe de clarifier les concepts.
Tous droits réservés @ C Département des relations industrielles de l’Université Laval, 1963
Ce document est protégé par la loi sur le droit d'auteur. L’utilisation des services d'Érudit (y compris la reproduction) est assujettie à sa politique d'utilisation que vous pouvez consulter en ligne.

https://apropos.erudit.org/fr/usagers/politique-dutilisation/ 


\section{INFORMATIONS}

\section{Socialisation et relations industrielles}

Nous publions ici les résumés des travaux qui ont été présentés lors du Congrès des relations industrielles de Laval en avril dernier. Les textes complets paraîtront sous peu dans un ouvrage intitulé Socialisation et relations industrielles.

\section{LA SOCIALISATION}

\section{GÉRARD Dion}

Quand on parle de «socialisation》 on suscite immédiatement des réactions diverses, selon la tournure d'esprit, les passions, les intérêts de ceux d̀ qui on s'adresse. C'est que le terme «socialisation》 recèle des ambiguités qui sont sources d'embarras et de confusions. Il importe de clarifier les concepts.

\section{NOTIONS DE SOCLALISATTON}

a) en psychologie ou psycho-sociologie:

Se réfère à l'homme dans ses rapports avec la société. C'est le processus par lequel la personnalité individuelle reconnaît son environnement et s'y adapte L'enfant doit se socialiser. Quand une personne ne réussit pas à se socialiser, on a affaire à un individu a-social.

b) en économique et en droit:

Se réfère aux choses, à leur propriété. C'est un régime juridique applícable à des biens. Secialisation signifie ici «nationalisation» ou « étatisation ».

\section{c) en sociologie:}

Se réfère à la fois aux hommes, aux biens et aux institutions. C'est un processus socio-culturel qui touche toute l'existence. C'est la tendance perpétuelle de l'homme vers une communication avec ses semblables, vers la coopération, vers l'association, et, au-dessus de tout, vers l'amitié. Cette tendance se réalise dans toutes les institutions de la vie sociale. Jean XXIII, dans Mater et Magistra, définit la socialisation comme étant «l'ensemble de ces interdépendances, chaque jour plus nombreuses, qui ont entraîné dans la vie et l'action des hommes de multiples formes de liens sociaux reconnus généralement en droit public ou privé ». Ces liens sociaux se traduisent en mille institutions, organisations, associations. 
a) Elle est un fait et non une doctrine. Ce fait n'est pas statique; il n'est pas un donné fini, achevé, mais il est un mouvement dynamique. En ce sens, il est autant une « tâche à accomplir qu'une réalité existentielle. Comme tel, il nécessite une doctrine, une politique. On devra le référer à une échelle de valeurs.

b) Elle n'est pas un fait nouveau. Son développement est parallèle à celui de la civilisation. Cependant, la socialisation contemporaine revêt un caractère particulier et se manifeste d'une manière différente.

c) Elle n'est pas seulement le résultat de liens sociaux naturels (liens familiaux, liens de voisinage immédiat), mais la conséquence de l'activité et la volonté des hommes, à la suite du développement des sciences et des techniques dans les domaines de la production et des communications; intensification des échanges matériels et culturels, multiplication des interdépendances.

d) Elle amène la prolifération encadrée de liens sociaux et de groupes pour répondre aux besoins fondamentaux de l'homme avec une diversification des institutions spécialisées. Abandon graduel de responsabilités à d'autres groupes, (vég. dans la famille: éducation, sécurité). On crée à plaisir des groupements nouveaux qui mettent en oeuvre chaque besoin, chaque idée.

e) Domination de l'esprit d'organisation. Tout est matière à construction sociale. Souci d'efficacité. Le monde social est de plus en plus le produit de cl'art », façonné par l'homme. Autrefois, on se laissait pousser par la tradition, la coutume, on acceptait les structures. Intervention de l'homme partout.

f) Conscience de l'unité. Tous les problèmes se situent par rapport à un ensemble plus vaste. Répercussions nationales et même internationales des situations ou des attitudes particulières.

g) Changement des lieux de décisions. Les objectifs poursuivis sont hors d'atteinte pour les possibilités d'action individuelle. Le monde ne peut être maitrisé que par des équipes: c'est une exigence de la technique et de la rationalisation.

h) Rôles de plus en plus divers du pouvoir politique qui doit, à son tour, posséder des connaissances techniques.

i) \& La socialisation n'est due en rien à l'impulsion aveugle des forces de la nature; elle est, au contraire, oeuvre de l'homme, être libre: sa nature le porte à agir, ne lui enlève pas la responsabilité de ses actes, bien qu'il doive reconnaître les lois de l'humanité en devenir et celles du mouvement de l'économie et ne puisse se soustraire entièremnt à l'influence du milieu ». (Jean XXIII, Mater et Magistra).

\section{SOCIALISATION ET ETATISATION}

La socialisation ne doit pas être confondue ni identifiée avec l'étatisation ou la propriété publique des moyens de production, quoique dans certains cas et 
dans certaines circonstances particulières, l'étatisation ou la nationalisation soit une forme normale et nécessaire de la socialisation.

\section{SOCIALISATION ET SOCLALISME}

Le mot \& socialisme > est aussi équivoque que celui de e socialisation s. On devrait parler des socialismes plutôt. Le socialisme est une doctrine. Divers groupements se réclament de cette doctrine dans leur action. Pour porter un jugement sur chacun de ces socialismes et chacun de ces groupements, il faut voir ce qui en est réellement, sans apriorisme. Le socialisme démocratique est aussi différent du socialisme marxiste qu'il l'est du libéralisme. Le socialisme de type anglo-saxon n'est pas le socialisme continental européen.

L'Eglise catholique a condamné le socialisme, mais elle a pris la peine de définir ce qu'elle entendait. On le trouve dans Mater et Magistra. Ce qu'il y a de condamné, c'est: a) une conception de vie close sur le temporel, dans laquelle le bien-être est considéré comme objectif suprême de la société; b) la poursuite d'une organisation sociale de la vie commune au seul niveau de la production, au grand préjudice de la vie humaine; c) en lui fait défaut tout principe de véritable autorité sociale. Or tous ces points sont condamnables dans toute doctrine qu'elle se qualifie de «socialiste ou de «capitaliste ». Aussi, les évêques du Canada ont-ils qualifié le genre de socialisme qu'ils condamnaient: le socialisme ematérialiste et < totalitaire $>$.

Entre le phénomène de socialisation et le socialisme considéré soit comme doctrine ou comme mouvement, les observateurs s'accordent à constater qu'il n'est point sûr qu'il existe des liens de causalité.

l.a socialisation, comme le soutiennent les évêques du Canada, peut être même une sauvegarde contre le socialisme matérialiste et totalitaire.

\section{AVANTAGES ET INCONVENIENTS DE LA SOCIALISATION}

Jusqu'ici le bilan de la socialisation comporte à la fois des avantages et des inconvénients.

Parmi les avantages, la socialisation a rendu possible, surtout dans le domaine économique et social, la satisfaction d'un grand nombre de droits de la personne humaine, entre autres ceux qui concernent les moyens d'existence, les soins médicaux, la diffusion et le progrès d'une culture de base, la formation professionnelle, le logement, le travail, un repos convenable et de sains loisirs. En outre grâce à la meilleure organisation des moyens modernes de diffusion de la pensée presse, cinéma, radio, télévision -, il est possible en tout lieu du monde, d'assister, pour ainsi dire, en personne, aux évènements mondiaux, si éloignés soient-ils s. (Mater et Magistra)

Parmi les inconvénients, \&la socialisation amplifie démesurément l'appareil bureaucratique, rend toujours plus minutieuse la réglementation juridique des rapports humains dans tous les secteurs de la vie en société et utilise des méthodes 
qui comportent un risque grave de ce que l'on caractérise aujourd'hui d'un mot «déshumanisation ». L'homme moderne voit se restreindre à l'excès, en bien des cas, la sphère dans laquelle il peut penser par lui-même, agir de sa propre initiative, exercer ses responsabilités, affirmer et enrichir sa personnalité ». (Card. Tardini).

La socialisation offre la possibilité pour plus de conformisme, de passivité, de paresse et d'automatisme en même temps qu'elle présente des chances pour l'éclosion de nouvelles libertés. La multiplicité des appartenances dégage l'homme de l'emprise d'un seul groupe. La multiplicité des groupes permet un équilibre de pouvoirs.

\section{POUR PARER AUX INCONVENIENTS DE LA SOCLAIISATION}

a) L'intervention de l'Etat est rendue plus nécessaire. Son rôle est de diriger, surveiller, coordonner, suppléer. Le rôle de suppléance n'est pas le premier. Il doit diriger d'une façon démocratique. Cette intervention doit s'accompagner d'une conscience aigue du bien commun tant chez les dirigeants politiques que chez les citoyens.

b) L'acceptation des groupements intermédiaires jouissant d'une réelle autonomie et poursuivant leurs objectifs dans la concorde et au bénéfice du bien commun.

c) Une participation consciente, active, responsable des individus à la vie et au fonctionnement des groupements. Cette participation doit être organisée à différents niveaux selon les capacités et les possibilités des individus.

\section{SOCLALISATION ET PLANIFICATION}

Dans le domaine économico-social, la planification n'est qu'un aspect de la socialisation. Il n'y a pas de doute que nous sommes engagés dans un mouvement irréversible de socialisation qui entraine une certaine planification. Nous pouvons accepter cette socialisation en restant aveugle et passif ou en être conscients et fouer un rôle actif. Nous n'avons pas le choix quant au mouvement lui-même. Tout ce que nous pouvons faire, c'est de fixer les objectifs, de choisir les moyens et de déterminer les agents d'exécution. Est-ce que cette socialisation sera effectuée d'une façon totalitaire ou bien sera-t-elle le résultat d'une coopération entre les intéressés à qui seront laissées les responsabilités qu'ils pourront assumer à différents niveaux? L'alternative est démocratie économique ou dictature totalitaire : il n'y a pas d'autre issue.

Alors une collaboration institutionnelle doit être organisée entre tous les agents de l'économie: les corps intermédiaires doivent coopérer entre eux et avec le gouvernement afin d'aider celui-ci à jouer un rôle positif dans l'économie pour le bien commun national et international.

Par groupements intermédiaires, nous entendons ici, syndicats ouvriers, associations d'employeurs, mais aussi tout groupe représentant les agents de l'économie 
à n'importe lequel niveau. Et l'objet de cette collaboration comprend sans restriction toutes les questions que les agents de l'économie ont en commun.

C'est cette coopération organisée entre les agents de la production, permettant et favorisant la participation de tous les intéressés pour le règlement de leurs propres affaires, avec la coopération du gouvernement pour la détermination des politiques économiques nationales et internationales à la lumière du bien commun que l'on désigne sous le nom d'une organisation démocratique de l'économie.

\section{CONCLUSION}

Même si l'on s'entend sur les objectifs à poursuivre, la mise sur pied d'une organisation démocratique de l'économie ne sera pas une tâche facile. Elle va demander d'abord un changement dans les esprits. Il va falloir cesser de se payer de mots, d'entretenir des \& mythes \$.

Ensuite, il sera indispensable de créer de nouvelles institutions et d'ajuster celles qui existent. On ne part pas du vide. On doit tenir compte des conditions dans lesquelles nous vivons, l'Amérique du Nord avec la division des pouvoirs entre le gouvernement fédéral et les gouvernements provinciaux; la législation actuelle; les caractéristiques de notre économie, sa dépendance des pays étrangers et spécialement des Etats-Unis; la structure et l'organisation des groupes représentant les agents de l'économie: associations d'employeurs, syndicats ouvriers; les besoins d'un pays qui avec sa géographie comprend diverses régions, divers marchés; l'existence de deux cultures, etc., etc.

Nous avons devant nous un défi difficile à rencontrer. Si la socialisation en vient à tyraniser qui que ce soit, la responsabilité n’en sera pas à la socialisation elle-même, mais à notre manque de volonté et d'imagination.

\section{L'ENTREPRISE FACE A LA SOCIALISATION}

RAYMOND GÉRIN Dans l'économie pré-industrielle, l'entreprise était généralement du type artisanal.

Dans cette entreprise composée d'un nombre restreint de travailleurs, l'instrument de production était l'outil personnel, le produit était fabriqué presqu'entièrement par le même ouvrier, le travail en série n'existait pratiquement pas. Enfin, la production se faisait sur commande c'est-à-dire que généralement on ne produisait pas à l'avance une certaine quantité de produits à être mis en vente par la suite. 